Line A presents a more complex puzzle yet to be solved.

Interstellar chemists have to use microwave ion spectroscopy in the interstellar medium to fill in the gaps as chemical models of the interstellar medium are poorly constrained. Synergistic observations of chemically related species are required to refine models of interstellar clouds and observations of ions are particularly important since ion-molecule reactions are a key synthetic process in the clouds. The $\mathrm{HCS}^{+}$ion is probably synthesized by reaction of formyl ion, $\mathrm{HCO}^{+}$, and ubiquitous $\mathrm{H}_{3}{ }^{+}$ion, with CS: $\mathrm{H}_{3}{ }^{+}$or $\mathrm{HCO}^{+}+\mathrm{CS} \rightarrow \mathrm{HCS}^{+}+\mathrm{H}_{2}$ or $\mathrm{CO}$. The major loss process for $\mathrm{HCS}^{+}$is recombination with electrons or reaction with $\mathrm{O}$ atoms: $\mathrm{HCS}^{+}+\mathrm{e}^{-} \rightarrow \mathrm{CS}+\mathrm{H}$ and
$\mathrm{HCS}^{+} \mathrm{O} \rightarrow \mathrm{OCS}^{+}+\mathrm{H}$. Simultaneous observations of $\mathrm{HCO}^{+}, \mathrm{HCS}^{+}, \mathrm{CO}$ and $\mathrm{CS}$ can therefore lead to constraints on the abundance of $\mathrm{H}_{3}{ }^{+}$, electrons and $\mathrm{O}$ atoms.

There are still scores of unidentified interstellar molecules and new ones are found as telescope sensitivities improve. The unscrambling of these signals via $a b$ initio calculations and clever observing techniques combined with more laboratory microwave ion spectroscopy will lead to more ion identifications. Many of the interstellar lines may turn out to be exotic highly reactive species difficult to produce on Earth but which persist for longer times in an environment where $10^{6}$ per $\mathrm{cm}^{3}$ is considered a dense region. The added challenge will be to confirm the identifications in Earth-based laboratories and to measure microwave transitions for other ions which might match up with lines already observed.

\footnotetext{
Thaddeus, P., Guelin, M. \& Linke, R. Astrophys. J. Lett. 246, I.41 (1981).

. Gudeman, C., Haese, N., Piltch, N. \& Woods, R. Astrophys. J. Lett. 246, L47 (1981).

3. Buhl, D. \& Snyder, L. Nature 228, 267 (1970).

4. Klemperer, W. Nature 227, 1230 (1970).

4. Klemperer, W. Nature 227, $1230(1970)$.
5. Herbst, E. \& Klemperer, W. Astrophys. J. 188, 255 (1974).

6. Snyder, L., Hollis, J., Ulich, B., Lovas, F. \& Buhi, D. BAAS 7 (no 3) 1I, 497 (1975).

7. Woods, G., Dixon, T., Saykally, R. \& Szanto, P. Phys. Rev. Lett. 35, 1269 (1975).

8. Dixon, T. \& Woods, C. Phys. Rev. Lett. 34, 61 (1975).

9. Erickson, N., Snell, R., Loren. R., Mundy, L. \& Plambeck, L. Astrophys. J. Lett. 205, L101 (1976).

Turner, B.E. Astrophys. J. Lett. 193, L83 (1974).

10. Turner, B.E. Astrophys. J. Lett. 193, L83 (1974).
11. Green, S., Montgomery, J. \& Thaddeus, P. Astrophys. J. Lett. 193, L89 (1976)

12. Saykally, R.J., Dixon, T.A., Anderson, T.G., Szanto, P.G. \& Woods, C.R. Astrophys, J. Lett. 205, 1.101 (1976).
}

\title{
Why study palaeoecology?
}

\section{from Andrew Hill}

As well as being the art of the soluble, science should be the art of the relevant. A distinction between these normally closely allied scientific activities seemed occasionally rather too evident at a colloquium on the environment of Plio-Pleistocene hominids held recently in Paris*.

Participants presented new information on a variety of fossil vertebrates and on fossil flora known from wood fragments and pollen. A few contributors arrived at more synthetic palaeoecological conclusions about fossil sites in eastern and southern Africa, using various techniques. There was even some mention of the hominids themselves, but only in the sense of placing the subject in some sort of general perspective. It was a little surprising to find practically no explicit discussion of why we might be interested in the environment of our Plio-Pleistocene ancestors; presumably a significant object is a better understanding of major human evolutionary shifts in terms of adaptation.

The main human morphological changes embody interesting evolutionary problems and it would have been valuable to try to explain them ecologically. One such problem is the origins of bipedalism, which these days might be equated with the divergence of hominids from their non-hominid ancestors. Another is dental change, presumably connected with changes in feeding, exemplified in its extreme form by the contrast between the Homo lineage and the very successful contemporary robust australopithecines. Yet another is the great increase in brain size during the evolution of Homo. How do all these relate to environmental and other ecological change in

*Colloque International sur l'Environnement des Hominidés au Plio-Pléistocène held in Paris at the end of June, under the auspices of the Fondation Singer-Polignac, was organized by Professor Yves Coppens, Director of the Muse de l'Homme. The proceedings of the conference will be published by the Foundation.
Africa during the Plio-Pleistocene?

That these clearly identifiable problems were not squarely tackled may reflect some major methodological difficulties with terrestrial vertebrate palaeoecology as currently practised. For example, information in palaeoecology is very sparse; even where it exists its time resolution or spatial relevance is not easy to assess. Consequently there is a justifiable tendency, as at this meeting, to believe in the primacy of data, to solve any problem solvable and to believe any scrap of evidence relevant to the specific problems about which we should be concerned. In contrast, in neoecological work data are more or less unlimited and workers recognize the need to be highly selective. They consider their main problems first and secondarily judge what features of the environment might be relevant to solving them. Clearly palaeoecology would benefit from a closer identification with its modern counterpart.

But a second problem comes from too close an imitation, for palaeoecologists readily accept and try to implement the techniques of neoecology. For example, the relative numbers of different species is often an important parameter of modern terrestrial communities and so people working on fossil vertebrates attempt to calculate the relative numbers of species in their assemblages. Unfortunately fossil assemblages are not like modern communities - the nature of the material is different and probably demands different and original approaches from those used in modern ecology. Instead of examining fossil collections with neoecological methods, we should probably be looking at them in their own terms, and referring to contemporary communities from this palaeoecological perspective.

Modern analogues of environment and species behaviour are also heavily relied upon. It seems so easy to say that the known fossil information is compatible with models reflecting modern environments that it becomes difficult to envisage ways in which past environments could differ from present ones. Do palaeoecologists even possess an adequate vocabulary to imagine anything other than savannah mosaic, more or less, wetter or drier, bush or grassland? Perhaps we need make more sophisticated distinctions if they are to have any chance of being relevant to the evolution of hominids and other creatures.

According to conventional criteria however, the meeting was a considerable success. The presentation and discussion of material possibly relevant to these more specific issues was very useful to those in the field. In addition to much new and basic taxonomic information, a biostratigraphical scheme was put forward for the important Lake Turkana sequence; relationships between east African sites and the less easily dateable sites in the south were clarified. It was resolved to collate various information for different localities and make this more generally available. The colloquium demonstrated that successful study of palaeoecology is extremely difficult, although the quality of the information collected shows that the situation is far from hopeless. However, palaeoecologists should stop sheltering behind assertions that the science is in a primitive 'data-gathering' stage; we need to get to grips with the relevant and interesting problems instead of assuming the more complacent and easy posture of acquiring more data just because it is possible to acquire it, of solving 'problems' just because they can be solved.

Andrew Hill is a Research Fellow at the National Museums of Kenya and at Yale University. 